\title{
EVALUACIÓN DE LA DIVERSIDAD DE ALGAS FITOPLANCTÓNICAS COMO INDICADORES DE LA CALIDAD DEL AGUA EN LAGUNAS ALTOANDINAS DEL DEPARTAMENTO DE PASCO (PERÚ)
}

\section{EVALUATION OF THE DIVERSITY OF PHYTOPLANKTONIC ALGAE AS WATER QUALITY INDICATORS IN HIGH ANDEAN LAKES OF THE DEPARTMENT OF PASCO (PERU)}

\author{
Maribel Baylón Coritoma ${ }^{1}$, Karen Roa Castro ${ }^{2}$, Tito Libio Sánchez ${ }^{3}$, Liliana Tapia Ugaz ${ }^{4}$, Enoc Jara Pena ${ }^{5}$, \\ Diego Macedo Prada ${ }^{6}$, Alan Salvatierra Sevillano ${ }^{7}$ y Abigail Dextre Rubina ${ }^{8}$
}

\begin{abstract}
Resumen
En el departamento de Pasco, Perú, la actividad minera constituye la principal actividad económica, generando hace décadas contaminantes ambientales como relaves mineros, aguas ácidas y emisiones provenientes de las minas, plantas concentradoras de metales y fundiciones, impactando negativamente los cuerpos de agua de cabecera de cuenca como lagunas Altoandinas. En el presente estudio se evaluó la calidad del agua de cinco lagunas (Lacsacocha, Yanamate, Quiulacocha, Milpo Andina y Huaroncocha), en base a la abundancia, riqueza y diversidad del fitoplancton. Así mismo, se evaluaron parámetros fisicoquímicos como $\mathrm{pH}$, conductividad, temperatura y metales pesados. Para la colecta de muestras se realizaron filtrados de $40 \mathrm{l}$ de agua en la orilla, mediante una red de fitoplancton de $10 \mu \mathrm{m}$. Los índices bióticos empleados para la evaluación de la calidad del agua fueron la diversidad de Shannon-Wiener (H') y el Índice Diatómico General (IDG). Se registraron 88 taxones distribuidos en siete phyla: Bacillariophyta (28), Charophyta (11), Chlorophyta (30), Cyanobacteria (15), Euglenophyta (2), Ochrophyta (1), Miozoa (1). El phylum más frecuente fue Bacillariophyta $(69.16 \%)$ seguido de Chlorophyta $(24.26 \%)$. Los índices bióticos permiten determinar que Yanamate, Lacsacocha, Quiulacocha y Milpo Andina presentaron aguas de mala calidad, mientras que el cuerpo de agua de la laguna Huaroncocha presentó calidad biológica óptima. Palabras clave: fitoplancton, indicadores de calidad, lagunas altoandinas, Pasco.
\end{abstract}

\begin{abstract}
In the Department of Pasco,Peru, mining activity has been the main economic activity for decades, having generated environmental pollutants such as mine tailings, acidic waters and smoke emissions from mines, concentration plants and foundries as well as negative impacts to high Andean lakes in the upper basins. The aim of this study was to evaluate the water quality of five high andean lakes (Lacsacocha, Yanamate, Quiulacocha, Milpo Andina and Huaroncocha) in the department of Pasco, based on phytoplankton abundance, richness and diversity. For the collection of samples, 401 of water were filtered on the shore, using a phytoplankton net of $10 \mu \mathrm{m}$. Four parameters were evaluated: $\mathrm{pH}$, conductivity, temperature and concentration of metals in the water. The biotic indexes used for water quality assessment were the Shannon-Wiener diversity $\left(\mathrm{H}^{\prime}\right)$ and the general diatomic index (GDI). 88 taxa were registered, distributed in seven phyla: Bacillariophyta (28), Charophyta (11), Chlorophyta (30), Cyanobacteria (15), Euglenophyta (2), Ochrophyta (1) and Miozoa (1). The most frequent phylum was Bacillariophyta (69.16\%), followed by Chlorophyta $(24.26 \%)$. Biotic indexes indicated that Yanamate, Lacsacocha, Quiulacocha and Milpo Andina were polluted while Huaroncocha waters had optimal biological quality.
\end{abstract}

Key words: phytoplankton, quality indicators, high Andean lakes, Pasco Department.

\section{Introducción.}

La Región de Pasco está situada en la parte central del Perú, en la vertiente oriental de la Cordillera de los Andes y su capital, Cerro de Pasco, es considerada como primera unidad minera en la producción de concentrados de zinc, plomo y plata en Sudamérica, y la cuarta productora de zinc en el mundo (Volcan,
2007). Existe una preocupación por la contaminación del agua en las cuencas de los ríos Alto Huallaga y San Juan y en la sub cuenca del río Tingo por la presencia de metales pesados, que afectan a la población de este entorno (Bianchini, 2009). Asimismo, el mayor impacto negativo se da en la utilización de lagunas 
como depósitos de relaves mineros, como se observa en las lagunas Yanamate y Quiulacocha.

Por otra parte, se conoce, que las comunidades fitoplanctónicas son muy sensibles a los cambios fisicoquímicos en su medio ambiente, por lo que muchas especies de fitoplancton son utilizadas como indicadoras de la calidad del agua (Reynolds, 1997; Reynolds et al., 2002; Brettum \& Andersen, 2005). Estos bioindicadores son útiles en los monitoreos ambientales en países industrializados (Streble \& Krauter, 1987). Por tanto, a partir de la observación de cambios en la composición y abundancia de especies, que ocurren en las comunidades acuáticas en el tiempo y espacio, se puede deducir el grado de contaminación (Yucra \& Tapia, 2008).

Las diatomeas son las microalgas más numerosas del fitobentos y fitoplancton; debido a sus características biológicas y ecológicas reflejan el estado ecológico de las aguas superficiales frente a impactos antrópicos (Cox, 1991). Una de las aplicaciones más comunes de la flora diatomológica dulceacuícola en el mundo es su uso como indicadores biológicos de la productividad, eutrofización, acidificación del medio y contaminación por metales pesados. Por tal razón, muchos especialistas como Pinilla (2000), Yucra (2005) Battarbee et al., 2010 y Calizaya-Anco et al. (2013), elaboraron diversos índices biológicos que usan la flora diatomológica. Además, las diatomeas han sido reconocidas y usadas mucho tiempo como indicadoras del $\mathrm{pH}$ del agua y actualmente son usadas en estudios de aguas acidificadas y ácidas (Battarbee et al., 2010). De igual forma, las cianobacterias son reconocidas como uno de los principales indicadores de eutrofización en cuerpos de agua dulce, ya que sus floraciones son frecuentes en las aguas afectadas por el enriquecimiento de nutrientes (Reynolds, 1984; Sondergaard \& Moss, 1998).

La eutrofización del agua dulce se considera como un problema de calidad de agua, que se traduce en el deterioro del medio acuático y en impactos en el consumo de agua. Al respecto Straskraba \& Tundisi (1999) señalan que la medición de la comunidad fitoplanctónica da información más precisa de los cambios en la calidad de agua, a diferencia de la variación en la concentración clorofila o de nutrientes. Pinilla (2000) recomienda contrastar la presencia y abundancia de las especies con otras variables fisicoquímicas, que permitan definir las condiciones del agua y el papel indicador de las especies.

En la actualidad, existen diversas medidas e índices para evaluar la calidad del agua, basada en elementos biológicos que permiten que el fitoplancton se relacione con la producción primaria, o asociaciones de algas y cianobacterias con las condiciones ambientales, incluyendo el nivel de degradación del lago o embalse. Estas herramientas de evaluación de la calidad se basan en datos de biomasa, composición, abundancia de fitoplancton y biovolumen (Hörnström, 1981; OCDE,
1982; Sládecek, 1973; Brettum, 1989; Barbe et al., 1990; Tremel, 1996; Reynolds et al., 2002; Brettum \& Andersen, 2005; Carvalho et al., 2007).

En nuestro país se han realizado estudios taxonómicos de las microalgas como bioindicadores de la calidad de agua, destacando los trabajos de: Montoya \& Espinosa (1985), quienes estudiaron a las microalgas de la Laguna de Oxidación de Ventanilla; Náquira (2006), quien evaluó a las Cianobacterias de los bofedales altoandinos de Candarave y su importancia en la calidad trófica de las aguas; Yucra \& Tapia (2008), utilizaron a las microalgas como bioindicadoras de polución orgánica en las brisas de Oquendo; Ortega et al. (2010), evaluaron la diversidad de la biota acuática y sus usos como indicadores ambientales en el Bajo Urubamba en donde incluyeron a las microalgas; Yucra (2005) y Calizaya-Anco et al. (2013), usaron a las diatomeas como bioindicadoras de calidad de agua.

El objetivo de este trabajo fue evaluar la calidad del agua en lagunas Altoandinas de la Región Pasco contaminadas con relaves mineros, tomando como referencia, la abundancia, riqueza y diversidad del fitoplancton como indicador alternativo de la calidad del agua.

\section{Materiales y métodos.}

\section{Área de estudio}

El muestreo se realizó en Octubre del 2015, en las principales lagunas de zonas Altoandinas de la Región de Pasco. Los cuerpos de agua evaluados fueron: Lacsacocha, Yanamate, Quiulacocha, Milpo Andina y Huaroncocha, georreferenciadas mediante el GPS (GARMIN eTrex ${ }^{\circledR}$ 30). Figura 1 y Tabla 1.

\section{Recolección de muestras}

Las muestras se colectaron realizando dos filtrados de 401 en cada laguna, con una red de fitoplancton de 10 micrómetros de abertura de malla y se procedió a la fijación directa empleando formaldehido al $4 \%$. Se obtuvieron un total de 14 muestras de fitoplancton en las cinco lagunas muestreadas, dos estaciones en el relave Milpo (M1 y M2) y la laguna Huaroncocha (H1 y H2) y una estación en las lagunas Lacsacocha (L-1), Yanamate (Y-1) y Quiulacocha (Q-1).

Determinación de metales pesados del agua

Los análisis de metales pesados en muestras de agua de las lagunas estudiadas fueron realizados en el Instituto de Corrosión y Protección de la Pontificia Universidad Católica del Perú (PUCP), en base al Método de Ensayo: "U.S. Environmental Protection Agency. 1994. Method 200.7, Revision 4.4: Determination of Metals and Trace Elements in Water and Wastes by Inductively Coupled Plasma-Atomic Emission Spectrometry".

Análisis de fitoplancton

Para el análisis cualitativo se tomó varias alícuotas de $1 \mathrm{ml}$ de la muestra, aproximadamente 20 gotas con pipeta Pasteur y se colocó en varios portaobjetos. Se 


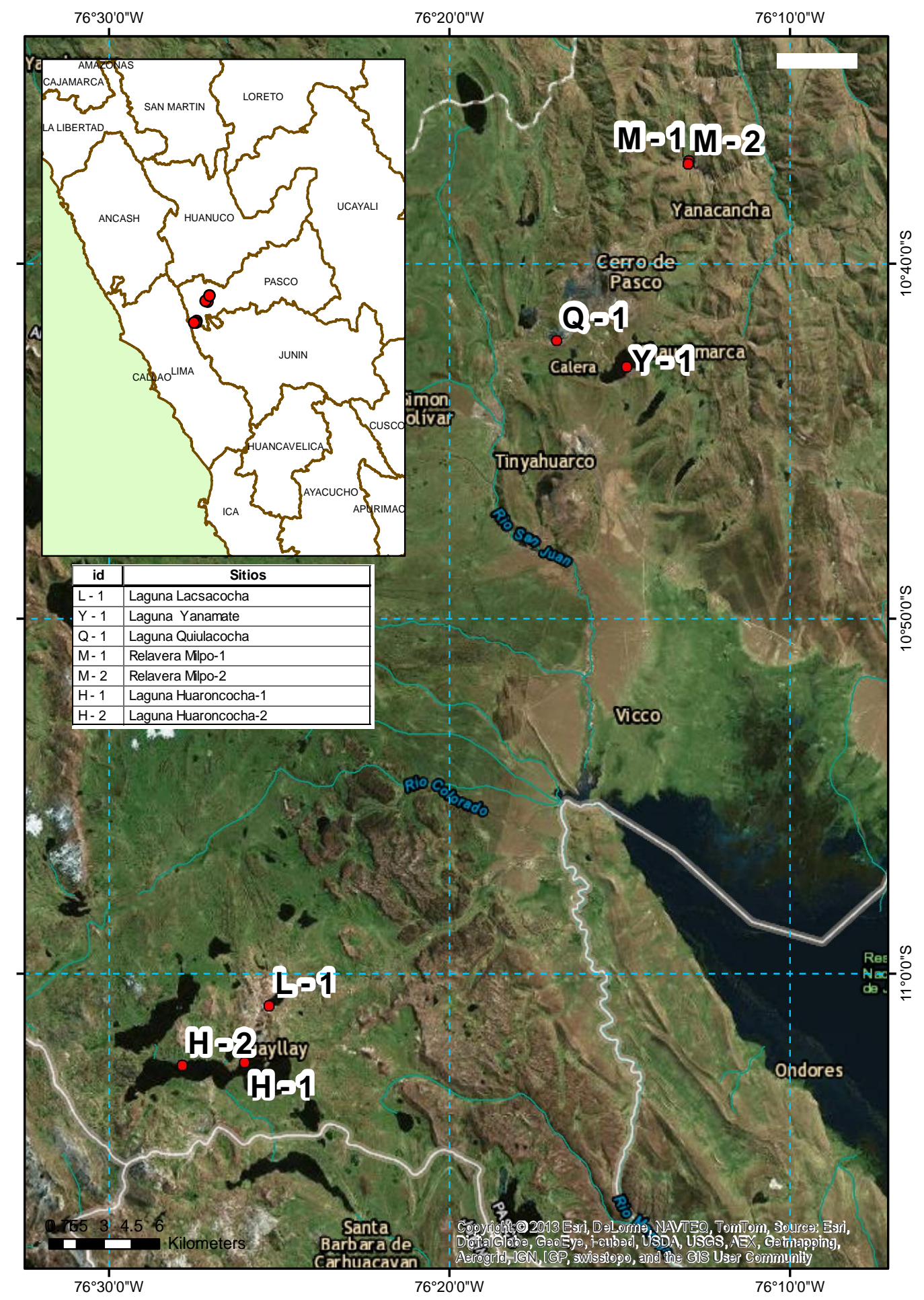

Figura 1. Mapa del área de estudio en donde se muestran las estaciones de muestreo de las lagunas Altoandinas estudiadas en la provincia de Pasco. Octubre del 2015.

llevó a un microscopio compuesto para su lectura e identificación con objetivos de 20X, 40X y 100X. Para la identificación de algunas cianobacterias y microalgas usamos diferentes técnicas de tinción como tinta china, lugol o azul de metileno; y para el caso particular de las diatomeas se procedió con la limpieza de las valvas mediante la destrucción de la materia orgánica (Método de Oxidación con Ácidos) según Battarbee (1986). Para el análisis cualitativo se anotaron todas las especies identificadas elaborando un listado de presencia 
Enero - Julio 2018

Tabla 1. Características y descripción de las lagunas Altoandinas estudiadas en la Provincia de Pasco. Octubre del 2015.

\begin{tabular}{|c|c|c|c|c|c|c|}
\hline ن & Lagunas & Distrito & $\begin{array}{l}\text { Coordenadas } \\
\text { Geográficas }\end{array}$ & $\begin{array}{r}\text { Altitud } \\
(\mathbf{m s n m})\end{array}$ & $\begin{array}{l}\text { Área } \\
\text { (Ha) }\end{array}$ & Descripción \\
\hline L-1 & Lacsacocha & Huayllay & $\begin{array}{l}11^{\circ} 0^{\prime} 54.18^{\prime \prime} \mathrm{S} \\
76^{\circ} 25^{\prime} 17.66^{\prime \prime} \mathrm{O}\end{array}$ & 4564 & 44.8 & $\begin{array}{l}\text { Laguna influenciada por restos de } \\
\text { los residuos sólidos de la minera } \\
\text { aledaña del lugar. }\end{array}$ \\
\hline $\mathrm{Y}-1$ & Yanamate & Chaupimarca & $\begin{array}{l}10^{\circ} 42^{\prime} 54.47^{\prime \prime} \mathrm{S} \\
76^{\circ} 14^{\prime} 48.79^{\prime \prime} \mathrm{O}\end{array}$ & 4347 & 180.0 & $\begin{array}{l}\text { Laguna empleada como depósito } \\
\text { de relave minero de la minera } \\
\text { aledaña del lugar. }\end{array}$ \\
\hline $\mathrm{Q}-1$ & Quiulacocha & Simón Bolivar & $\begin{array}{l}10^{\circ} 42^{\prime} 9.65^{\prime \prime ~ S} \\
76^{\circ} 16^{\prime} 51.36^{\prime \prime} \mathrm{O}\end{array}$ & 4273 & 114.0 & $\begin{array}{l}\text { Laguna empleada como depósito } \\
\text { de relave minero de la minera } \\
\text { aledaña del lugar. }\end{array}$ \\
\hline$M-1$ & Milpo Andina-1 & Yanacancha & $\begin{array}{l}10^{\circ} 37^{\prime} 5.79^{\prime \prime} \mathrm{S} \\
76^{\circ} 12^{\prime} 59.37^{\prime \prime} \mathrm{O}\end{array}$ & 3903 & 11.0 & $\begin{array}{l}\text { Zona aledaña a la tubería de los } \\
\text { restos de residuos de la minera del } \\
\text { lugar }\end{array}$ \\
\hline M - 2 & Milpo Andina-2 & Yanacancha & $\begin{array}{l}10^{\circ} 37^{\prime} 10.89^{\prime \prime} \mathrm{S} \\
76^{\circ} 13^{\prime} 0.95^{\prime \prime} \mathrm{O}\end{array}$ & 3900 & 11.0 & $\begin{array}{l}\text { Zona de influencia directa de la } \\
\text { descarga de los residuos de la } \\
\text { minera aledaña del lugar. }\end{array}$ \\
\hline $\mathrm{H}-1$ & Huaroncocha-1 & Huayllay & $\begin{array}{l}11^{\circ} 2^{\prime} 29.77^{\prime \prime S} \\
76^{\circ} 26^{\prime} 1.04 " \mathrm{O}\end{array}$ & 4555 & 1058.0 & Zona asociada a macrófitas. \\
\hline $\mathrm{H}-2$ & Huaroncocha-2 & Huayllay & $\begin{array}{l}11^{\circ} 2^{\prime} 35.33^{\prime \prime ~ S} \\
76^{\circ} 27^{\prime} 50.97^{\prime \prime} \mathrm{O}\end{array}$ & 4564 & 1058.0 & $\begin{array}{l}\text { Zona asociada a macrófitas con } \\
\text { abundante vegetación ribereña y } \\
\text { presencia de bofedales. }\end{array}$ \\
\hline
\end{tabular}

(1)/ausencia (0) de la población fitoplanctónica. En la identificación de las especies se emplearon los trabajos de Komárek \& Anagnostidis (1999), Komárek \& Anagnostidis (2005), Komárek (2013) Streble \& Krauter (1987), Whitford \& Schumacher (1973), Prescott (1983), West et al. (1991), John et al. (2002), Wehr et al. (2015).

Para el análisis cuantitativo, se tomó $1 \mathrm{ml}$ de muestra previamente homogeneizada y se trasvasó a la cámara de conteo de Sedgewick-Rafter para su lectura en un microscopio invertido marca, de acuerdo a las normas de los APHA (2012). Los resultados de abundancia del fitoplancton fueron expresados en Ind.1 ${ }^{1}$ (individuos/litro). Se calculó el índice de diversidad Shannon y Wiener ( $\left.\mathrm{H}^{\prime}\right)$ en base $\log _{2}$, la Riqueza de Margalef (d) y la Dominancia de Simpson (D). Debido a que las diatomeas resultaron estar representadas en todas las estaciones de muestreo, se calculó el Índice Diatómico General (IDG), índice que sólo requiere realizar determinaciones a nivel de género ya que su aplicación nos ayuda a determinar la calidad ecológica de los cuerpos de agua.

$\underline{\text { Relación entre el fitoplancton y los parámetros }}$ fisicoquímicos

Se relacionó el fitoplancton con los parámetros fisicoquímicos, a partir de la similaridad de Bray-Curtis para las muestras de fitoplancton y de las distancias euclidianas con los datos fisicoquímicos, empleando el método de correlación de Spearman entre las matrices de similaridad biótica y abiótica (Clarke y Warwick, 1994; Clarke y Goley, 2001). Para este propósito se utilizó el análisis "Biota and/or Environment Matching” BIOENV del programa PRIMER v6.

\section{Resultados. \\ Composición de Fitoplancton}

Con los resultados del análisis cualitativo de las lagunas Lacsacocha, Yanamate Quiulacocha, Milpo y la Laguna Huaroncocha se han registrado 88 taxones reunidos en siete Phylum (Ver Tabla 2 en Anexos). El phylum Chlorophyta presentó la mayor riqueza específica, con un $34.1 \%$ del total de especies, seguida de Bacillariophyta con un $31.88 \%$ del total de especies, siendo la clase Bacillariophyceae la de mayor aporte a este porcentaje. Cyanobacteria, Charophyta, Miozoa, Ochrophyta y Euglenophyta presentaron menores valores.

Abundancia de Fitoplancton

La densidad de fitoplancton total varió entre los taxa. El Phylum Bacillariophyta fue el más representativo con 15973 Ind. $^{-1}(81.38 \%)$ seguido de Chlorophyta con 2317 Ind. $^{-1} \quad(11.80 \%) \quad$ y Cyanobacteria con 1098 Ind. $^{-1}$ (5.59\%), entre los grupos con menor densidad se encuentran Charophyta con 132 Ind. $^{-1}(0.67 \%)$, Euglenophyta y Miozoa con 2 Ind. $1^{-1}(0.01 \%)$ cada uno. La densidad total del fitoplancton por lagunas fue mayor en la relavera Milpo Andina (8 981 - 4795 Ind.1 $^{-1}$ ), seguido de la laguna Huaroncocha (2 848 Ind. $^{-1}$ ) y menor en la laguna Lacsacocha (55 Ind. - $^{-1}$ ) (Ver Tabla 3 en Anexos).

Evaluación de los Parámetros fisicoquímicos del agua

La conductividad más baja se encontró en Huaroncocha con $110 \mu \mathrm{S} . \mathrm{cm}^{-1}$ y la más alta en Yanamate con $5200 \mu \mathrm{S} . \mathrm{cm}^{-1}$. La temperatura fluctuó entre $10.6^{\circ} \mathrm{C}$ en Yanamate y $19^{\circ} \mathrm{C}$ en Huaroncocha. El pH más bajo se encontró en Yanamate (2.4) y el más alto Huaroncocha (8.11). Los valores de conductividad para Milpo (M-2), Quiulacocha y Yanamate están por 
Tabla 4. Parámetros Fisicoquímicos de las lagunas altoandinas de la Provincia de Pasco. Octubre del 2015. (Color rojo: valores que superan los LMP).

\begin{tabular}{llllllll}
\hline & L-1 & Y-1 & Q-1 & M-1 & M-2 & H-1 & H-2 \\
\hline Conductividad $\left(\boldsymbol{\mu S} / \mathbf{c m}^{-1}\right)$ & 880 & 5200 & 3860 & 420 & 1600 & 130 & 110 \\
pH & 7.28 & 2.4 & 2.49 & 5.6 & 6.4 & 7.11 & 8.11 \\
Temperatura $\left({ }^{\circ} \mathbf{C}\right)$ & 13.7 & 10.6 & 13 & 11.4 & 14 & 19 & 17 \\
\hline
\end{tabular}

encima de los Límites Máximos Permisibles $(\leq 1000$ $\left.\mu \mathrm{S} . \mathrm{cm}^{-1}\right)$ de los Estándares Nacionales de Calidad Ambiental (ECA) para el agua: Categoría 4 (Conservación del Ambiente Acuático) aprobados por Decreto Supremo No 015-2015-MINAM. Con respecto al potencial de hidrógeno $(\mathrm{pH})$ Yanamate, Quiulacocha y Milpo (M-2) presentaron valores bajos de $\mathrm{pH}$ cercanos a 2, los cuales no cumplen con los ECA (Tabla 4).

Metales Pesados determinados en el agua

En las lagunas Lacsacocha Yanamate, Milpo y Quiulacocha, los valores de metales pesados como Cadmio, Arsénico, Zinc, Cobre y Plomo, están por encima de los ECAs (Cuerpos de agua categoría 4). No se puede determinar si la presencia de otros metales analizados como Mercurio, Selenio y Talio está dentro de los ECA que establece la normativa peruana. Debido a que los Límites de detección son menores a los limites detectados por el Instituto de Corrosión de la PUCP, en todo caso si hubiese contaminación, sería baja. Asimismo, los metales analizados están por debajo del rango detectado por el método de análisis (U.S. Environmental Protection Agency, 1994) empleado por la PUCP (Tabla 5).

Índices de diversidad

La mayor diversidad y equitabilidad de especies se encontró en la Laguna Huaroncocha (H-1 y H-2) y la laguna Milpo Andina (M-1) y la menor diversidad se encontró en la laguna Yanamate (Y-1) debido a la alta dominancia de especies que presentó (Tabla 6).

Índice Diatómico General (IDG)

Coste \& Ayphassorho (1991), indica que el IDG se determina por 3 variables: la sensibilidad a la polución de cada especie, la amplitud ecológica y su abundancia dentro de la muestra. Los valores de IDG se miden en el intervalo desde 1 hasta 5 , en orden decreciente según los niveles de contaminación. Se observa que los IDG, van de 2.71 (Contaminación fuerte) a 4.61 (calidad biológica óptima) (Tabla 7).

Relación entre el fitoplancton y los parámetros fisicoquímicos

La conductividad eléctrica presentó alta correlación (0.74) con la comunidad fitoplanctónica en los cinco ambientes lenticos, seguido del $\mathrm{pH}(0.55)$ y por último la temperatura (0.51). El BIOENV mostro un $\mathrm{R}=0.76$ con un porcentaje de significancia del $4 \%(\mathrm{p}<0.05)$.

\section{Discusión.}

Se encontró la mayor riqueza de fitoplancton en la laguna Huaroncocha (H-1 con 45 especies), dominando el Phylum Chlorophyta con $46.8 \%$ en donde destacó la clase Chlorophyceae (Sphaerocystis sp., Crucigeniella cf. rectangularis, Scenedesmus sp., Pandorina sp. etc.) y el Phylum Bacillariophyta con $46.4 \%$, siendo la clase Bacillariophyceae la más representativa (Epithemia sorex, Encyonema sp., Fragilaria sp., etc.; Figura 2 en Anexos). La alta riqueza de especies podría deberse a la variedad de hábitats presentes en la laguna, plantas acuáticas sumergidas $\mathrm{y}$ emergentes, condiciones fisicoquímicas favorables y aguas con presencia de metales pesados dentro de los límites permisibles señalados según los Estándares Nacionales de Calidad Ambiental (ECA) D.S. $\quad \mathrm{N}^{\circ}$ 015-2015-MINAM. Montoya \& Espinosa (1985), mencionan que la conversión de la materia orgánica de los desechos a sales inorgánicas, nitratos, fosfatos y sulfatos fácilmente absorbidos por las algas y otras plantas macrófitas, originan un significante incremento de su biomasa que se refleja en la alta capacidad fotosintética de lagos y lagunas.

La menor riqueza de fitoplancton se encontró en Yanamate (6 especies), probablemente debido a la contaminación que presenta esta relavera, la cual ha sido utilizada para el vertimiento de residuos y aguas

Tabla 5. Resultados del análisis de metales pesados (ppm) en muestras de agua de las Lagunas Altoandinas evaluadas en la Provincia de Pasco (Color rojo: valores que superan los LMP).

\begin{tabular}{lllllll}
\hline Metales $\left(\mathrm{mg}^{-1}\right)$ & Lacsacocha & Yanamate & Milpo & Quilacocha & Huaroncocha & $\begin{array}{l}\text { Límite Máximo } \\
\text { Permisible }\end{array}$ \\
\hline Arsénico & 0.057 & 12.7 & 0.069 & 3.3 & 0.034 & $\mathbf{0 . 1 5}$ \\
Cadmio & 0.006 & 0.5 & 0.008 & 0.175 & N.D. & $\mathbf{0 . 0 0 0 2 5}$ \\
Cinc & 0.982 & 152 & 1.139 & 62 & 0.136 & $\mathbf{0 . 1 2}$ \\
Cobre & 0.3 & 26.5 & 0.083 & 3.4 & 0.012 & $\mathbf{0 . 1}$ \\
Mercurio & $<\mathbf{0 . 0 1 0}$ & $<\mathbf{0 . 0 1 0}$ & $<\mathbf{0 . 0 1 0}$ & $<\mathbf{0 . 0 1 0}$ & $<\mathbf{0 . 0 1 0}$ & $\mathbf{0 . 0 0 0 1}$ \\
Niquel & $<0.013$ & 0.073 & 0.016 & 0.042 & N.D. & $\mathbf{0 . 0 5 2}$ \\
Plomo & 0.3 & 0.217 & 0.6 & 0.174 & $<\mathbf{0 . 0 5 8}$ & $\mathbf{0 . 0 0 2 5}$ \\
Selenio & $<\mathbf{0 . 0 1 0}$ & $<\mathbf{0 . 0 1 0}$ & $<\mathbf{0 . 0 1 0}$ & $<\mathbf{0 . 0 1 0}$ & $<\mathbf{0 . 0 1 0}$ & $\mathbf{0 . 0 0 5}$ \\
Talio & $<\mathbf{0 . 0 1 6}$ & $<\mathbf{0 . 0 1 6}$ & $<\mathbf{0 . 0 1 6}$ & $<\mathbf{0 . 0 1 6}$ & $<\mathbf{0 . 0 1 6}$ & $\mathbf{0 . 0 0 0 8}$ \\
\hline
\end{tabular}

Información comparada con los ECAs del Decreto Supremo $\mathbf{N}^{\circ}$ 015-2015-MINAM y los resultados de las muestras de agua analizadas por el Instituto de Corrosión y Protección, Pontificia Universidad Católica del Perú. 
Tabla 6. Índices de Diversidad, equidad y dominancia de las microalgas fitoplanctónicas de las lagunas altoandinas en la Región Pasco. Octubre del 2015.

\begin{tabular}{cccc}
\hline Estaciones & $\begin{array}{c}\text { Diversidad de } \\
\text { Shannon- } \\
\text { Wiener }\left(\mathbf{H}^{\prime}\right)\end{array}$ & $\begin{array}{c}\text { Equitabilidad } \\
\text { de Pielou }(\mathbf{J})\end{array}$ & $\begin{array}{c}\text { Dominancia } \\
\text { de Simpson } \\
(\mathbf{D})\end{array}$ \\
\hline L-1 & 2.77 & 0.83 & 0.21 \\
Y-1 & 1.95 & 0.75 & 0.33 \\
Q-1 & 2.87 & 0.80 & 0.21 \\
M-1 & 2.91 & 0.55 & 0.22 \\
M-2 & 1.22 & 0.26 & 0.58 \\
H-1 & 3.17 & 0.59 & 0.16 \\
H-2 & 3.36 & 0.72 & 0.18 \\
\hline
\end{tabular}

ácidas provenientes de la unidad de producción de Cerro de Pasco desde 1981 (Volcan, 2000), superando los ECAs. para metales pesados. La abundancia fitoplanctónica se encontró dominada por las diatomeas pennadas de pequeño tamaño $(66.67 \%)$ como Pinnularia sp., Navicula sp. y Nitzschia sp. Además, de la Chlorophyta Radiofilum sp. (26.64\%), especie cosmopolita eurihalina que se encuentra frecuentemente en aguas blandas y ácidas (John, 2011). Quiulacocha también presentó una menor riqueza (20 especies), cuya especie dominante fue Nitzschia sp. $(41.35 \%)$, al igual en el caso anterior, es muy probable que este resultado se deba a la contaminación que presenta esta laguna por metales pesados, ya que estuvo siendo utilizada como un relave minero desde el año 1900 hasta 1976 por la empresa norteamericana Cooper Corporation (Bianchini, 2009).

La conductividad eléctrica más baja se observó en Huaroncocha $\left(<130 \mu \mathrm{S} . \mathrm{cm}^{-1}\right)$, lo que evidenciaría bajos niveles de iones metálicos en la laguna, en Lacsacocha $\left(880 \mu{\mathrm{S} . \mathrm{cm}^{-1}}^{-}\right)$, la estación M-2 de Milpo (1 $600 \mu \mathrm{S} . \mathrm{cm}^{-}$ $\left.{ }^{1}\right)$ y Quiulacocha $\left(3860 \mu \mathrm{S} . \mathrm{cm}^{-1}\right)$ tiende a aumentar estos valores. Además, el valor más alto fue encontrado en Yanamate $\left(5200 \mu \mathrm{S} . \mathrm{cm}^{-1}\right)$. Según estas mediciones, estas lagunas no cumplen con los Estándares Nacionales de Calidad Ambiental (ECA) para el agua: Categoría 4 (Conservación del Ambiente Acuático) aprobados por Decreto Supremo No 015-2015-MINAM $\left(\leq 1 \quad 000 \mu \mathrm{S} . \mathrm{cm}^{-1}\right)$ indicando la presencia de

Tabla 7. Índice Diatómico General (IDG) de las lagunas Altoandinas de la Provincia de Pasco. Octubre del 2015.

\begin{tabular}{|c|c|c|}
\hline Laguna & IDG & Significado \\
\hline Lacsacocha & 3.71 & $\begin{array}{l}\text { Contaminación moderada. } \\
\text { Eutrofización. }\end{array}$ \\
\hline Yanamate & 3.79 & $\begin{array}{l}\text { Contaminación moderada. } \\
\text { Eutrofización. }\end{array}$ \\
\hline Quiulacocha & 2.71 & $\begin{array}{l}\text { Desaparición de especies } \\
\text { sensibles. Contaminación } \\
\text { fuerte. }\end{array}$ \\
\hline Milpo Andina & 2.87 & $\begin{array}{l}\text { Desaparición de especies } \\
\text { sensibles. Contaminación } \\
\text { fuerte. }\end{array}$ \\
\hline Huaroncocha & 4.61 & Calidad biológica óptima. \\
\hline
\end{tabular}

contaminantes metálicos y de sales disueltas. En la Relavera Milpo se presentaron dos zonas bien diferenciadas, la estación M-1 sin influencia directa de los desechos inorgánicos y alejada de los vertimientos de la minera presentó baja conductividad $\left(420 \mu{\mathrm{S} . \mathrm{cm}^{-}}^{-}\right.$ 1) y la estación M-2 directamente impactada por los residuos o desechos mineros presentó una conductividad más alta $\left(1600 \mu \mathrm{S} . \mathrm{cm}^{-1}\right)$. Con respecto al potencial de Hidrógeno, Yanamate, Quiulacocha presentaron valores bajos de $\mathrm{pH}$ cercanos a 2, Milpo (M-2) presento $\mathrm{pH}$ 6.4, debido al tratamiento alcalino que tienen los efluentes, antes de ser emitidos al cuerpo receptor. Estos cuerpos de agua no cumplen con los Estándares Nacionales de Calidad Ambiental (ECA) para el agua: Categoría 4 (6.5-9.0). Para H-1 y H-2 los valores de $\mathrm{pH}$ fueron $\geq 7.11$, cumpliendo con los ECA.

En nuestro estudio, se observó que la Relavera Milpo Andina presentó densidad moderada de diatomeas como, Nitzschia spp. (889 a 6563 org. $1^{-1}$ ) Achnanthes sp. (1 875 a 1950 org. $1^{-1}$ ) Pinnularia sp. (625 og. $\left.\mathrm{l}^{-1}\right)$ y Navicula sp. (114 org. $\left.\mathrm{l}^{-1}\right)$. La alta concentración de los metales Cadmio, Zinc, y Plomo en el agua no afectó la densidad de estas especies. Nuestros resultados muestran que la contaminación alta por metales como Cadmio, Arsénico, Zinc, Cobre y Plomo afectan la riqueza y diversidad de las comunidades fitoplanctónicas en las relaveras Lacsacocha, Yanamate y Quiulacocha, pero no afectan significativamente la densidad de las diatomeas pequeñas como Navicula sp. (188 org. $1^{-1}$ ), Nitzschia sp. (113 org. $\mathrm{l}^{-1}$ ) y Pinnularia sp. (825 org. $\mathrm{l}^{-1}$ ), encontradas en Yanamate y Nitzschia sp. (43 org. $\left.1^{-1}\right)$ en Quiulacocha. Cattaneo et al. (2011), estudió la respuesta de las diatomeas litorales a la contaminación por metales en 11 lagos situados a diferentes distancias de la fundición minera Rouyn-Noranda (Quebec), encontrando que las diatomeas responden a altas concentraciones de metales de tres maneras: (i) disminución o erradicación de los taxa (ii) no se afectan, y (iii) taxas que prosperan con la presencia de metales. La contaminación por metales $(\mathrm{Cd}, \mathrm{Hg}$ y $\mathrm{Cu})$ no afectó la densidad de las diatomeas; Fragilaria construens var. venter, Fragilaria construens var. pumila y Brachysira vítrea, más se incrementó la abundancia relativa con la contaminación por metales. En cambio en nuestro estudio la contaminación por metales afectó la presencia de especies de la Familia Fragilariaceae, ya que se observó una total ausencia de estos taxa en Lacsacocha, Yanamate, Quiulacocha y Milpo.

El análisis del índice de diversidad de Shannon Wienner (Lobo \& Kobayasi, 1990), engloba la riqueza de especies y sus componentes, como índice general de diversidad, además este índice también determina el estado de contaminación de una masa de agua, considerado así como un buen indicador del impacto que ejerce el ambiente sobre las especies. Se presenta una escala de valores normales de diversidad para cada 
nivel de contaminación que van de 0 a 4 bits.ind ${ }^{-1}$, en donde los valores mayores que 3 indican agua limpia; 1-3 indican contaminación moderada y valores inferiores a 1 se caracterizan por estar fuertemente contaminada (Wilham \& Dorris, 1968). Teniendo en cuenta esta escala, las relaveras Lacsacocha, Yanamate, Quiulacocha y Milpo $\left(1.22-2.91\right.$ bits.ind. $\left.^{-1}\right)$ presentaron una contaminación moderada, la baja diversidad de especies se debe a la composición fitoplanctónica dominada por Bacillariophyta, relacionadas a $\mathrm{pH}$ ácidos, bajos niveles de conductividad y metales pesados por debajo de los ECAs. En la laguna Huaroncocha (>3.17 bits.ind..$\left.^{-1}\right)$ es clara la presencia de aguas limpias, evidenciando una alta diversidad de especies por la presencia de varios filo como Bacillariophyta, Charophyta, Chlorophyta, Cyanobacteria, Euglenophyta y Ochrophyta. En la laguna Huaroncocha las Bacillariophyta más abundantes fueron Encyonema sp. Epithemia sorex, Gomphonema sp. y Cocconeis placentula, especies cosmopolitas (Taylor et al., 2007) seguido de las Chlorophyta como Sphaerocystis sp. Crucigeniella cf. rectangularis Scenedesmus sp. y Oocystis cf. lacustris, especies probablemente cosmopolitas y comunes en comunidades planctónicas (John, 2011) y Charophyta como Closterium cf. lúnula y Elakatothrix sp. especies cosmopolitas, planctónicas y mundialmente distribuida en diferentes habitas (Brook \& Johnson, 2011). El crecimiento de estas comunidades fitoplanctónicas ha sido favorecido por las condiciones ambientales óptimas como luz, temperatura y $\mathrm{pH}$, además estas aguas no presentaron contaminación por metales, en consecuencia, favoreció el crecimiento plantónico de varios filos.

Las diatomeas son sensibles a los cambios en la calidad del agua, sus ciclos de vida son rápidos siendo capaces de colonizar rápidamente los sustratos y reaccionar con rapidez a las perturbaciones ambientales. Por este motivo se analizó el Índice Diatómico General (IDG), índice que sólo requiere realizar determinaciones a nivel de género. Para este estudio se contó con 22 taxones para determinar su aplicación. El IDG es un índice bastante complejo en cuanto a los datos requeridos, se basa en respuestas a diferentes grados de contaminación, a la adaptabilidad ecológica de los individuos y a su abundancia en el medio muestreado (Rivas et al., 2010). Los valores de IDG para Huaroncocha (4.61) nos indicó que el agua tiene una calidad biológica óptima y normal, en cambio Lacsacocha, Yanamate, Quiulacocha y Milpo (1.89 3.79) indican contaminación fuerte a moderada. Huaroncocha presentó una dominancia de diatomeas pennales y centrales de mediano tamaño como Encyonema sp. Epithemia sorex, Gomphonema sp. Cocconeis placentula, Fragilariaceae, Nitzschia sigmoidea etc., en cambio Lacsacocha, Yanamate, Quiulacocha y Milpo una dominancia de diatomeas pequeñas tolerantes a la contaminación como Nitzschia sp. Navicula sp. y Pinnularia sp. Lange-Bertalot (1979) menciona que el género Nitzschia sp. no solo puede persistir en ambientes altamente contaminados sino que también su reproducción puede verse estimulada.

Las correlaciones de Spearman obtenidas del BIOENV indican que la variable conductividad eléctrica alcanzó una alta correlación (0.74) con la comunidad de fitoplanctónica, seguido del $\mathrm{pH}(0.55)$ y la temperatura (0.51). Según Celis et al. (2008) la conductividad eléctrica influye en la distribución, composición, biomasa y densidad del fitoplancton. Las relaveras Yanamate y Quiulacocha presentaron los valores más altos de conductividad y más bajos de $\mathrm{pH}$, variables que podrían haber ocasionado una erradicación de las diatomeas de mediano tamaño de los géneros Encyonema sp. Epithemia sp., Gomphonema sp., Cocconeis sp. y Fragilaria sp. Blinn \& Bailey (2001) mencionan que los aumentos de iones disueltos afectan la abundancia y la riqueza de las diatomeas y Battarbee et al. (2010), señalan que las diatomeas planctónicas en lagos ácidos a menudo están pobremente desarrolladas y en los lagos muy ácido y acidificados a menudo carecen totalmente de diatomeas planctónicas. Las abundancias y riquezas de los taxones de fitoplancton en cada laguna estudiada si se encuentran afectadas por las variables fisicoquímicas propias de cada ambiente estudiado (Fontúrbel \& Castaño, 2011).

\section{Conclusiones.}

La contaminación por metales puede afectar la riqueza y diversidad de las comunidades fitoplanctónicas en las relaveras Lacsacocha, Yanamate y Quiulacocha, pero no afectan significativamente la densidad de pequeñas diatomeas. Las variables conductividad eléctrica y $\mathrm{pH}$ han afectado la composición y abundancia de las diatomeas planctónicas en las lagunas Yanamate y Quiulacocha.

El índice de diversidad de Shannon Wienner evidencia una contaminación moderada para las relaveras; Lacsacocha, Yanamate, Quiulacocha y Milpo, dada por la dominancia de Bacillariophyta, la laguna Huaroncocha presentó aguas limpias, evidenciando la presencia de varios filo como Bacillariophyta, Charophyta, Chlorophyta, Cyanobacteria, Euglenophyta y Ochrophyta.

Los valores de IDG para Huaroncocha nos indicaron que el agua tiene una calidad biológica óptima, en cambio Lacsacocha, Yanamate, Quiulacocha y Milpo indicaron contaminación fuerte a moderada. Huaroncocha presentó una dominancia de diatomeas Pennales y Centrales de mediano tamaño en cambio las relaveras Lacsacocha, Yanamate, Quiulacocha y Milpo una dominancia de diatomeas pequeñas tolerantes a la contaminación como el género Nitzschia sp.

Milpo, Quiulacocha y Yanamate no cumplieron con los Estándares Nacionales de Calidad Ambiental 
(ECA) para conductividad y pH en el agua: Categoría 4 (Conservación del Ambiente Acuático). Si bien, la conductividad y $\mathrm{pH}$ nos pueden indicar la calidad del agua, los valores de metales pesados como Cadmio, Arsénico, Zinc, Cobre y Plomo, nos permitió concluir la presencia de contaminación en las relaveras Lacsacocha, Yanamate, Quiulacocha y Milpo y el buen estado de la calidad del agua de la laguna Huaroncocha.

\section{Agradecimientos.}

Este estudio se realizó dentro de las actividades del Proyecto FINCyT "Monitoreo y evaluación de la calidad del Agua en cabecera de cuenca del río Rímac, lagos y lagunas de la región central del Perú para determinar organismos indicadores y marcadores moleculares de contaminación por metales pesados". Convenio $\quad \mathrm{N}^{\circ}$ 391-PNICP Innóvate Perú. Agradecimiento a José Zavala por la elaboración del mapa.

\section{Literatura citada.}

APHA (American Public Health Association) 2012. Standard Methods for the examination of water and Wasterwater. $22^{\text {nd }}$. American Public Health Association, American Water Works Association, Water Environment Federation.

Barbe J., Lavergne E., Rofes G., Lascombe M., Rivas J., Bornard C.H. \& De Benedittis J. 1990. Diagnose rapide de plans d'eau. Informations Techniques du CEMAGREF, 79:1-8.

Battarbee R.W., Charles D. F., Dixit S.S. \& Renberg I. 2010. Diatoms as indicators of surface water acidity. Publisher: Cambridge University https://doi.org/10.1017/CBO9780511613005.006

Battarbee R.W. 1986. Diatom analysis. 527-570 pp.

Bianchini F. 2009. Evaluación de la Calidad de los Recursos Hídricos en la Provincia de Pasco y de la Salud en el Centro Poblado de Paragsha. Asociación Civil Centro de Cultura Popular Labor.

Blinn D.W. \& Bailey P.C.E. 2001. Land-use influence on stream water quality and diatom communities in Victoria, Australia: a response to secondary salinization. Hydrobiologia 466: 231-244.

Brettum P. 1989. Alger som indikatorer pa vannkvalitet I norske innsjoer. Planteplankton. NIVA Report, 111pp.

Brettum P. \& Andersen T. 2005. The use of phytoplankton as indicators of wáter quality. NIVA Report, 197pp.

Brook A.J. \& Johnson L. R. 2011. Order Zygnematales. In: The freshwater algal flora of the British Isles. An identification guide to freshwater and terrestrial algae. Second edition. (John, D.M., Whitton, B.A. \& Brook, A.J. Eds), pp. 524-554. Cambridge: Cambridge University Press.

Calizaya-Anco J., Avendaño M. \& Delgado I. 2013. Evaluación de la calidad del agua fluvial con diatomeas (Bacillariophyceae), una experiencia en Tacna, Perú. Revista Peruana de Medicina Experimental y Salud Pública, 30(1), 58-63. DOI: http://dx.doi.org/10.1590/S1726-46342013000100012
Carvalho L., Dudley B., Dodkins I., Clarke R., Jones J., Thackeray S. \& Maberly S. 2007. Phytoplankton Classification Tool (Phase 2). Final report. Edinburgh, SNIFFER (Scotland \& Northern Ireland Forum for Environmental Research), 94pp. (CEH Project Number: C03236).

Cattaneo A., Couillard Y., Wunsam S. \& Fortin C. 2011. Littoral diatoms as indicators of recent water and sediment contamination by metals in lakes. Journal of Environmental Monitoring. Mar; 13(3):572-582. http://dx.doi.org/ 10.1039/c0em00328.

Celis S., Mancera E. \& Leon J. 2008. Evaluación de la Rotirerofauna presente en el complejo de Pajarales durante la época lluviosa, departamento del Magdalena, Colombia. Acta Biologica Colombiana, 13(2),23-40.

Clarke K.R \& Warwick R.M. 1994. Change in Marine Communities. An approach to statistical analysis and interpretation. Plymouth Marine Laboratory. Inglaterra.

Clarke K. \& Goley R. 2001.Plymout routines in multivariate ecological research v5. User manual/tutorial. PRIMER-E LTDA. 91pp.

Coste M. \& Ayphassorho H. 1991. Etude de la qualité des eaux du bassin Artois Picardie à l'aide des communautés de diatomées benthiques (Application des indices diatomiques). Raport Cemagref. Bordeaux-Agence de 1'Eau Artois-Picardie, Douai, 277.

Cox E.J. 1991. What is the basis for using diatoms as monitors of river quality. Use of algae for monitoring rivers. Institut für Botanik, Universität in Innsbruck, 3340.

Decreto Supremo N015-2015-MINAM. Estándares Nacionales de la Calidad Ambiental para el Agua.

Fontúrbel F. \& Castaño G. 2011. Relationships between Nutrient Enrichment and the Phytoplankton Community at an Andean Oligotrophic Lake: A multivariate Assessment. Ecología Aplicada 10(2): 75-81.

Hörnström E. 1981. Trophic characterization of lakes by means of qualitative phytoplankton analysis. Limnologica (Berlin) 13, 249-261.

John D.M., Whitton B.A. \& Brook A.J. 2002. The freshwater algal flora of the British Isles: an identification guide to freshwater and terrestrial algae (Vol. 1). Cambridge University Press. doi: 10.5860/choice.40-3394. http://dx.doi.org/10.5860/choice.40-3394.

John D.M. 2011. Phylum Chlorophyta. Orders Chaetophorales, Microsporales, Ulotrichales. In: The freshwater algal flora of the British Isles. An identification guide to freshwater and terrestrial algae. Second edition. (John, D.M., Whitton, B.A. \& Brook, A.J. Eds), pp. 524-554. Cambridge: Cambridge University Press.

Komárek J. 2013. Cyanoprokaryota - 3. Teil / 3rd Part: Heterocytous Genera. - In: Büdel B., Gärtner G., Krienitz L. \& Schagerl M. (eds.), Süswasserflora von Mitteleuropa (Freshwater Flora of Central Europe), Springer Spektrum Berlin, Heidelberg, 1130pp.

Komárek J. \& Anagnostidis K. 1999. Cyanoprokaryota - 1. Teil: Chroococcales. En: ETTL, Hanuš, GÄRTNER, Georg, HEYNING, Hermann y MOLLENHAUER, Dieter. Süsswasserflora von Mitteleuropa. Jena: Gustav Fischer. Vol. 19, 1era parte, p. 1-548. 
Komárek J. \& Anagnostidis K. 2005. Cyanoprokaryota-2. Teil/ 2nd Part: Oscillatoriales. In: Büdel B., Krienitz L., Gärtner G. \& Schagerl M. (eds.). Süsswasserflora von Mitteleuropa 19/2, Elsevier/Spektrum, Heidelberg, 759 pp.

Lange-Bertalot H. 1979. Pollution tolerance of diatoms as a criterion for water quality estimation. Nova Hedwigia Beiheft. 64:285-304.

Lobo E. \& Kobayasi H. 1990. Shannon's diversity index applied to some freshwater diatom assemblages in the Sakawa River System (Kanagawa Pref., Japan) and its use as an indicator of water quality. Jpn J Phycol (Sorui). 38: $229-43$.

Montoya H. \& Espinosa J. 1985. Algas de la Laguna de Oxidación de Ventanilla, Callao, Perú. Boletín de Lima, 42: 41-68.

Náquira T. 2006. Cianobacterias de los bofedales altoandinos de Candarave y su importancia en el biomonitoreo de la calidad trófica de las aguas. Tesis para optar el título profesional de Biólogo Microbiólogo. Universidad Nacional Jorge Basadre Grohmann.

OCDE 1982. Eutrophication of Waters, Assessment and Control. Organisation for Economic Cooperation and Development. Paris, 154 pp.

Ortega H., Chocano L., Palma C. \& Samanez I. 2010. Biota acuática en la Amazonía Peruana: diversidad y usos como indicadores ambientales en el Bajo Urubamba (Cusco Ucayali). Rev. Perú. Biol. 17:1. DOI: http://dx.doi.org/10.15381/rpb.v17i1.47

Pinilla G. 2000. Indicadores Biológicos en Ecosistemas Acuáticos Continentales de Colombia. Compilación Bibliográfica. Fundación Universidad de Bogotá Jorge Tadeo Lozano. Centro de Investigaciones Científicas. Santafé de Bogotá, Colombia.

Prescott G.W. 1983. How to know the Freshwater Algae, third ed. W.C. Brown, Iowa, 293p.

Reynolds C.S. 1984. The Ecology of Freshwater Phytoplankton. Cambridge University Press.

Reynolds C.S. 1997. Vegetation processes in the Pelagic: a model for ecosystem theory. Excellence in Ecology.

Reynolds C., Huszar V., Kruk C., Flores N.L. \& Mel S. 2002. Towards a functional classification of the freshwater phytoplankton. Journal of Plankton Research, 24(5), 417428.
Sládecek V. 1973. System of water quality from the Biological point of view. Arch. Hydrobiol. 7: 1-218.

Straskraba M. \& Tundisi J.G. 1999. Reservoir Water Quality Management, Guidelines of Lake.

Streble H. \& Krauter D. 1987. Atlas de los microorganismos de agua dulce. La vida en una gota de agua. Ediciones Omega, S.A. Barcelona, España. 372 p.

Sondergaard M. \& Moss B. 1998. Impact of submerged macrophytes on phytoplankton in shallow freshwater lakes. Ecological Studies, 131, 115-132.

Taylor J.C., Harding W.R. \& Archibald C.G.M. 2007. An illustrated guide to some common diatom species from South Africa. Water Research Commission, WRC report TT 282/07.

Tremel B. 1996. "Determination of the trophic state by qualitative and quantitative phytoplankton analysis in twi gravel pit lakes" Hydrobiologia 323:1-38.

U.S. Environmental Protection Agency. 1994. Method 200.7, Revision 4.4: Determination of Metals and Trace Elements in Water and Wastes by Inductively Coupled Plasma-Atomic Emission Spectrometry.

Volcan Compañía Minera S.A.A. Memoria Anual 2007.

West J., Round F.E., Crawford R.M. \& Mann D.G. 1991. The Diatoms: Biology \& Morphology of the Genera. Taxon, 40(1), $\quad 156 . \quad$ Doi:10.2307/1222958. http://dx.doi.org/10.2307/1222958

Wehr J.D., Sheath R.G. \& Kociolek J.P.(Eds.) 2015. Freshwater algae of North America: ecology and classification. Elsevier. doi:10.1016/c2010-0-66664-8. http://dx.doi.org/10.1016/c2010-0-66664-8

Whitford L.A. \& Schumacher G.J. 1973. A Manual of FreshWater Algae. Sparks Press, Raleigh, N.C. 321 pp.

Yucra H.A. 2005. Uso de Diatomeas como bioindicadoras de calidad de agua. The Biologist (Lima), 3: 5 .

Yucra H.A. \& Tapia P.M. 2008. El uso de microalgas como bioindicadoras de polución orgánica en brisas de Oquendo. Callao, Perú. Biologist (Lima), 6:1.

Wilham J.L. \& Dorris T.C. 1968. Biological parameters of water quality criteria, Bioscience, 18, 447-481. 


\section{ANEXOS}

Tabla 2. Tabla de riqueza específica de cianobacterias y microalgas encontradas en las lagunas altoandinas estudiadas en la Provincia de Pasco. Octubre 2015.

\begin{tabular}{|c|c|c|c|c|c|c|c|}
\hline \multirow[t]{2}{*}{ ESPECIES } & \multicolumn{7}{|c|}{ Sitios de muestreo } \\
\hline & L-1 & Y-1 & Q-1 & M-1 & M-2 & HU-1 & HU- \\
\hline & RIA & & & & & & \\
\hline Chroococcales & & & & 1 & & 1 & \\
\hline Aphanothece sp. & & & & 1 & & & \\
\hline Johannesbaptistia sp. & & & & 1 & & & \\
\hline Gomphosphaeria sp. & & & & & 1 & & 1 \\
\hline Oscillatoriales & 1 & & & 1 & 1 & 1 & \\
\hline Borzia sp. & & & & & 1 & 1 & 1 \\
\hline Oscillatoria sp. & & & 1 & 1 & 1 & 1 & \\
\hline Lyngbya sp. & & & & 1 & & & \\
\hline Phormidium sp. & & & & 1 & 1 & 1 & 1 \\
\hline Anabaena sp. & & & 1 & 1 & 1 & 1 & 1 \\
\hline Heteroleibleinia sp. & & & & & & & 1 \\
\hline Leptolyngbya sp. & & & & & & & 1 \\
\hline Planktolyngbya sp & & & & 1 & & & \\
\hline Pseudanabaena sp. & & & & 1 & 1 & 1 & 1 \\
\hline Pseudanabaenaceae & & & & 1 & & & 1 \\
\hline
\end{tabular}

PHYLUM: BACILLARIOPHYTA

Clase: Bacillariophyceae

Achnanthes sp.

Achnanthidium sp.

Asterionella formosa Hassall

Cocconeis placentula Ehrenberg

Cymbella sp. 1

Cymbella sp. 2

Diatoma sp.

Encyonema sp.

Epithemia adnata (Kützing) Brébisson

Epithemia sorex Kützing

Gomphonema acuminatum Ehrenberg

Gomphonema truncatum Ehrenberg

Gomphonema sp.

Navicula sp. 1

Nitzschia cf. linearis

Nitzschia sigmoidea (Nitzsch) W.Smith

Nitzschia sp. 1

Nitzschia sp. 2

Stauroneis sp.

Pinnularia sp.

Rhopalodia sp.

Ulnaria ulna (Nitzsch) Compère

Clase: Coscinodiscophyceae

Melosira varians C.Agardh

Clase: Fragilariophyceae

Fragilaria sp.

Fragilariaceae

MICROALGAS

1

1

1

1

Fragilaria crotonensis Kitton

Clase: Mediophyceae

Cyclotella sp.

Discotella sp.

PHYLUM: CHAROPHYTA

Closterium cf. lunula

Closterium sp.

Cosmarium cf. contractum

Cosmarium cf. botrytis

\begin{tabular}{|c|c|c|c|c|c|c|}
\hline & & 1 & 1 & 1 & & \\
\hline & & & 1 & & & \\
\hline & & & 1 & & & \\
\hline & & & 1 & & 1 & 1 \\
\hline & & 1 & 1 & & 1 & \\
\hline 1 & & & & & & \\
\hline & 1 & 1 & & & & \\
\hline & & & 1 & & 1 & 1 \\
\hline 1 & & 1 & & & & 1 \\
\hline & & & 1 & 1 & 1 & 1 \\
\hline & & & 1 & 1 & 1 & \\
\hline & & & 1 & 1 & 1 & 1 \\
\hline & & & & 1 & 1 & 1 \\
\hline 1 & 1 & 1 & 1 & 1 & & \\
\hline & & & & 1 & & \\
\hline 1 & & & 1 & 1 & 1 & 1 \\
\hline 1 & 1 & 1 & 1 & 1 & 1 & 1 \\
\hline & & & 1 & 1 & & \\
\hline & & 1 & & & & \\
\hline 1 & 1 & 1 & 1 & 1 & 1 & 1 \\
\hline & & 1 & & & & \\
\hline & & 1 & 1 & 1 & & \\
\hline 1 & & 1 & & 1 & & 1 \\
\hline & & & 1 & & & \\
\hline 1 & & & & 1 & & 1 \\
\hline & & & & & 1 & \\
\hline & & 1 & & & & 1 \\
\hline & 1 & & & & & \\
\hline & & & & & 1 & \\
\hline & & & & & 1 & 1 \\
\hline & & & 1 & & & \\
\hline & & & & & 1 & 1 \\
\hline
\end{tabular}


Tabla 2. Tabla de riqueza específica de cianobacterias y microalgas encontradas en las lagunas altoandinas estudiadas en la Provincia de Pasco. Octubre 2015.

\begin{tabular}{|c|c|c|c|c|c|c|c|}
\hline \multirow[t]{2}{*}{ ESPECIES } & \multicolumn{7}{|c|}{ Sitios de muestreo } \\
\hline & L-1 & Y-1 & Q-1 & M-1 & M-2 & HU-1 & HU-2 \\
\hline Cosmarium sp. & & & & & & 1 & \\
\hline Staurastrum cf. furcigerum & 1 & & & & & & \\
\hline Staurastrum sp. & & & 1 & 1 & & 1 & \\
\hline Mougeotia sp. 1 & 1 & & & 1 & 1 & 1 & 1 \\
\hline Mougeotia sp. 2 & & & & & 1 & & \\
\hline Spirogyra sp. & & & & & 1 & & \\
\hline Elakatothrix sp. & & & & & & 1 & 1 \\
\hline \multicolumn{8}{|l|}{ PHYLUM: CHLOROPHYTA } \\
\hline Chlamydomonadales & & & & & 1 & 1 & 1 \\
\hline Palmella sp. & & & & & & & 1 \\
\hline Sphaerocystis sp. & & & & & 1 & 1 & 1 \\
\hline Eudorina sp. & & & & & & 1 & \\
\hline Pandorina morum (O.F.Müller) Bory & & & & & & 1 & \\
\hline Tetrasporaceae & & & & & & 1 & \\
\hline Oedogonium sp. & 1 & & 1 & 1 & & 1 & 1 \\
\hline Ankyra sp. & & & & & & & 1 \\
\hline Pediastrum boryanum (Turpin) Meneghini & & & 1 & 1 & 1 & 1 & 1 \\
\hline Pediastrum duplex Meyen & & & & 1 & & 1 & 1 \\
\hline Tetraedron minimum (A.Braun) Hansgirg & & & & 1 & & & \\
\hline Tetraedron sp. & & & & & & 1 & \\
\hline Microspora sp. & & & & & & 1 & \\
\hline Coelastrum cf. microporum & & & & 1 & 1 & & \\
\hline Comasiella arcuata Lemmermann & & & & 1 & & & \\
\hline Desmodesmus cf. maximus & & & & & 1 & 1 & \\
\hline Desmodesmus sp. & & & & & & & 1 \\
\hline Scenedesmus sp. & & & & 1 & 1 & 1 & 1 \\
\hline Scenedesmus cf. bijuga & & & & & & & 1 \\
\hline Westella sp. & & & & & 1 & & \\
\hline Radiofilum sp. & & 1 & & & & & \\
\hline Ankistrodesmus cf. falcatus & & & & 1 & & & \\
\hline Dictyosphaerium sp. & & & & & 1 & 1 & \\
\hline Crucigeniella cf. rectangularis & & & & 1 & 1 & 1 & 1 \\
\hline Lagerheimia cf. citriformis & & & & & 1 & & \\
\hline Lagerheimia sp. & & & & & 1 & & \\
\hline Oocystis cf. lacustris & & & & & & 1 & 1 \\
\hline Oocystis solitaria Wittrock & & & & & & 1 & \\
\hline Ulotrichales & 1 & & 1 & 1 & 1 & & 1 \\
\hline Ulothrix sp. & 1 & & 1 & 1 & 1 & 1 & \\
\hline \multicolumn{8}{|l|}{ PHYLUM: MIOZOA } \\
\hline Peridinium cf. cinctum & & & & & & 1 & \\
\hline \multicolumn{8}{|l|}{ PHYLUM: OCHROPHYTA } \\
\hline Tribonema sp. & & & 1 & 1 & 1 & 1 & \\
\hline \multicolumn{8}{|l|}{ PHYLUM: EUGLENOPHYTA } \\
\hline Euglena sp. & & & & & & & 1 \\
\hline Trachelomonas sp. & & & & & & 1 & \\
\hline
\end{tabular}


Tabla 3. Tabla de la composición y abundancia de cianobacterias y microalgas de las lagunas altoandinas estudiadas en la Provincia de Pasco. Octubre del 2015.

\begin{tabular}{|c|c|c|c|c|c|}
\hline TAXAS & Lacsacocha & Yanamate & $\begin{array}{l}\text { LAGUNAS } \\
\text { Quiulacocha }\end{array}$ & Milpo Andina & Huaroncocha \\
\hline Bacillariophyta & 22 & 1239 & 74 & 12562 & 2076 \\
\hline Nitzschia cf. linearis & 0 & 0 & 0 & 263 & 0 \\
\hline Nitzschia sigmoidea & 5 & 0 & 0 & 4 & 12 \\
\hline Nitzschia sp. 1 & 0 & 113 & 43 & 7369 & 115 \\
\hline Nitzschia sp. 2 & 0 & 0 & 0 & 83 & 0 \\
\hline Cocconeis placentula & 0 & 0 & 0 & 6 & 13 \\
\hline Cymbella sp. 1 & 5 & 0 & 8 & 17 & 0 \\
\hline Encyonema sp. & 0 & 0 & 0 & 81 & 801 \\
\hline Gomphonema acuminatum & 0 & 0 & 0 & 4 & 4 \\
\hline Gomphonema truncatum & 0 & 0 & 0 & 0 & 4 \\
\hline Gomphonema sp. & 0 & 0 & 0 & 11 & 100 \\
\hline Fragilariaceae & 0 & 0 & 0 & 0 & 34 \\
\hline Fragilaria crotonensis & 0 & 0 & 0 & 0 & 11 \\
\hline Ulnaria ulna & 0 & 0 & 4 & 21 & 0 \\
\hline Asterionella formosa & 0 & 0 & 0 & 99 & 0 \\
\hline Diatoma sp. & 0 & 38 & 0 & 0 & 0 \\
\hline Achnanthes sp. & 0 & 0 & 8 & 3825 & 0 \\
\hline Navicula sp. 1 & 0 & 188 & 2 & 116 & 0 \\
\hline Pinnularia sp. & 10 & 825 & 9 & 657 & 14 \\
\hline Epithemia cf. sorex & 0 & 0 & 0 & 6 & 951 \\
\hline Melosira varians & 2 & 0 & 0 & 0 & 15 \\
\hline Cyclotella sp. & 0 & 0 & 0 & 0 & 2 \\
\hline Discotella $\mathrm{sp}$. & 0 & 75 & 0 & 0 & 0 \\
\hline Charophyta & 6 & $\mathbf{0}$ & 2 & 26 & 98 \\
\hline Closterium cf. lunula & 0 & 0 & 0 & 0 & 53 \\
\hline Closterium sp. & 0 & 0 & 0 & 0 & 4 \\
\hline Cosmarium cf. botrytis & 0 & 0 & 0 & 0 & 2 \\
\hline Cosmarium cf. contractum & 0 & 0 & 0 & 2 & 0 \\
\hline Staurastrum cf. furcigerum & 2 & 0 & 0 & 0 & 0 \\
\hline Staurastrum sp. & 0 & 0 & 2 & 2 & 16 \\
\hline Mougeotia sp. 1 & 4 & 0 & 0 & 11 & 0 \\
\hline Mougeotia sp. 3 & 0 & 0 & 0 & 11 & 0 \\
\hline Elakatothrix sp. & 0 & 0 & 0 & 0 & 23 \\
\hline Chlorophyta & 47 & 450 & 12 & 263 & 1545 \\
\hline Chlamydomonadales & 0 & 0 & 0 & 38 & 84 \\
\hline Palmella sp. & 0 & 0 & 0 & 0 & 6 \\
\hline Sphaerocystis sp. & 0 & 0 & 0 & 9 & 604 \\
\hline Eudorina sp. & 0 & 0 & 0 & 0 & 2 \\
\hline Pandorina sp. & 0 & 0 & 0 & 0 & 71 \\
\hline Oedogonium sp. & 4 & 0 & 2 & 15 & 38 \\
\hline Ankyra sp. & 0 & 0 & 0 & 0 & 4 \\
\hline Pediastrum boryanum & 5 & 0 & 8 & 15 & 0 \\
\hline Pediastrum duplex & 0 & 0 & 0 & 4 & 13 \\
\hline Tetraedron minimum & 0 & 0 & 0 & 2 & 8 \\
\hline Microspora sp. & 0 & 0 & 0 & 0 & 2 \\
\hline Coelastrum cf. microporum & 0 & 0 & 0 & 53 & 0 \\
\hline Comasiella arcuata & 0 & 0 & 0 & 4 & 0 \\
\hline Desmodesmus cf. maximus & 0 & 0 & 0 & 2 & 2 \\
\hline Scenedesmus sp. & 0 & 0 & 0 & 4 & 128 \\
\hline Scenedesmus cf. bijuga & 0 & 0 & 0 & 0 & 4 \\
\hline Westella sp. & 0 & 0 & 0 & 2 & 0 \\
\hline Radiofilum sp. & 0 & 450 & 0 & 0 & 0 \\
\hline Ankistrodesmus cf. falcatus & 0 & 0 & 0 & 4 & 0 \\
\hline Dictyosphaerium sp. & 0 & 0 & 0 & 2 & 2 \\
\hline Crucigeniella $\mathrm{cf}$. rectangularis & 0 & 0 & 0 & 11 & 475 \\
\hline Lagerheimia cf. citriformis & 0 & 0 & 0 & 13 & 0 \\
\hline Lagerheimia sp. & 0 & 0 & 0 & 11 & 0 \\
\hline Oocystis cf. lacustris & 0 & 0 & 0 & 0 & 69 \\
\hline Oocystis solitaria & 0 & 0 & 0 & 0 & 8 \\
\hline
\end{tabular}


Tabla 3. Tabla de la composición y abundancia de cianobacterias y microalgas de las lagunas altoandinas estudiadas en la Provincia de Pasco. Octubre del 2015.

\begin{tabular}{|c|c|c|c|c|c|}
\hline \multirow[t]{2}{*}{ TAXAS } & \multicolumn{5}{|c|}{ LAGUNAS } \\
\hline & Lacsacocha & Yanamate & Quiulacocha & Milpo Andina & Huaroncocha \\
\hline Ulotrichales & 8 & 0 & 0 & 8 & 0 \\
\hline Ulothrix sp. & 30 & 0 & 2 & 6 & 4 \\
\hline Cyanobacteria & $\mathbf{0}$ & $\mathbf{0}$ & 8 & 835 & 255 \\
\hline Chroococcales & 0 & 0 & 0 & 60 & 21 \\
\hline Aphanothece sp. & 0 & 0 & 0 & 96 & 0 \\
\hline Johannesbaptistia sp. & 0 & 0 & 0 & 4 & 0 \\
\hline Oscillatoriales & 0 & 0 & 0 & 38 & 2 \\
\hline Borzia sp. & 0 & 0 & 0 & 4 & 118 \\
\hline Oscillatoria sp. & 0 & 0 & 8 & 11 & 6 \\
\hline Lyngbya sp. & 0 & 0 & 0 & 2 & 0 \\
\hline Phormidium sp. & 0 & 0 & 0 & 25 & 6 \\
\hline Anabaena sp. & 0 & 0 & 0 & 8 & 21 \\
\hline Nostoc sp. & 0 & 0 & 0 & 28 & 0 \\
\hline Heteroleibleinia sp. & 0 & 0 & 0 & 0 & 62 \\
\hline Leptolyngbya sp. & 0 & 0 & 0 & 0 & 36 \\
\hline Planktolyngbya $\mathrm{sp}$ & 0 & 0 & 0 & 126 & 0 \\
\hline Pseudanabaena sp. & 0 & 0 & 0 & 493 & 4 \\
\hline Miozoa & $\mathbf{0}$ & $\mathbf{0}$ & $\mathbf{0}$ & o & 2 \\
\hline Peridinium cf. cinctum & 0 & 0 & 0 & 0 & 2 \\
\hline Ochrophyta & $\mathbf{0}$ & $\mathbf{0}$ & 8 & 90 & 6 \\
\hline Tribonema sp. & 0 & 0 & 8 & 90 & 6 \\
\hline Euglenophyta & $\mathbf{0}$ & $\mathbf{0}$ & $\mathbf{0}$ & $\mathbf{0}$ & 2 \\
\hline Trachelomonas sp. & 0 & 0 & 0 & 0 & 2 \\
\hline TOTAL & 75 & 1689 & 104 & 13776 & 3984 \\
\hline
\end{tabular}



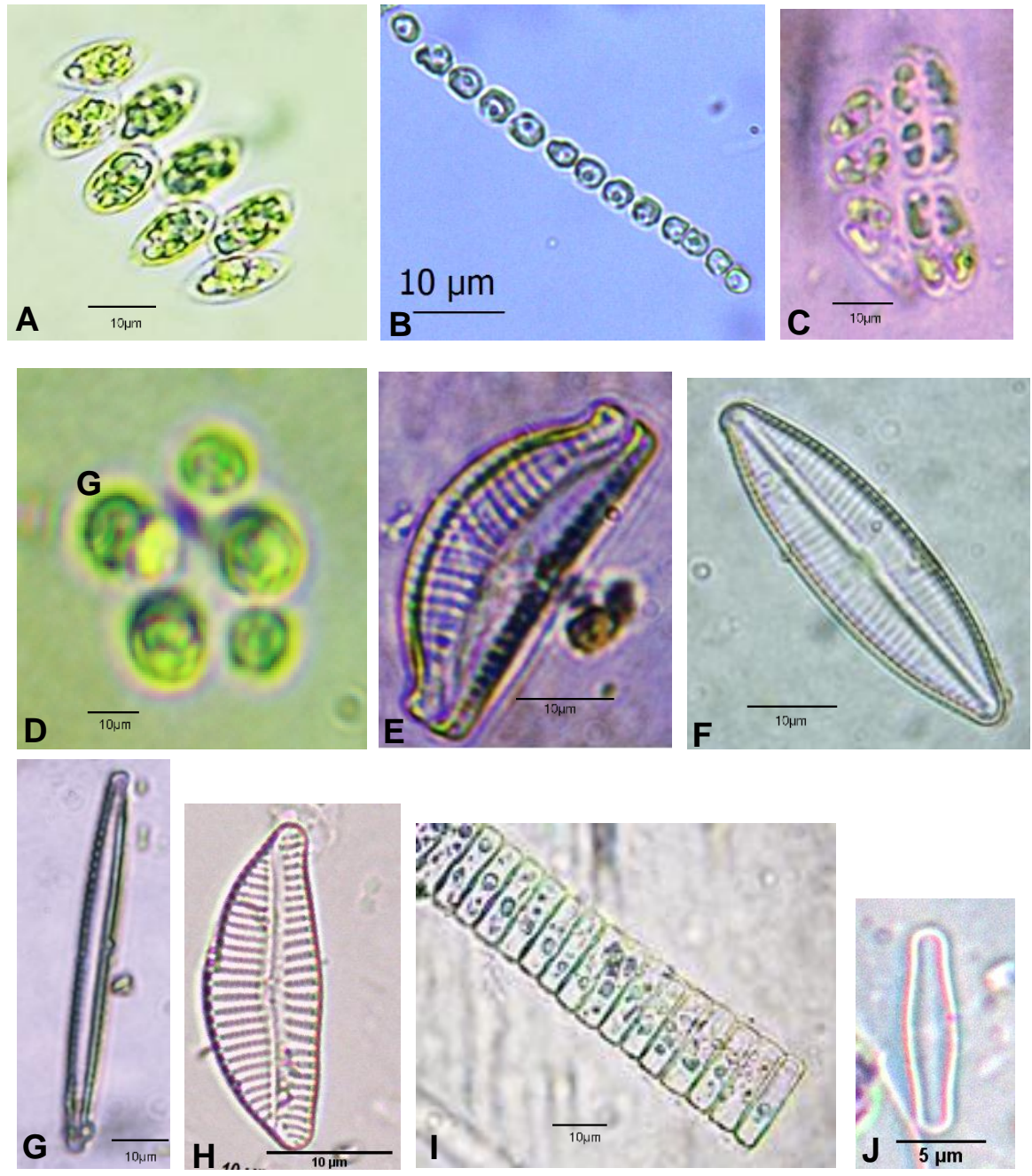

Figura 2. Microfotografías de algunas microalgas identificadas en los ambientes lénticos altoandinos evaluados del departamento de Pasco. A. Scenedesmus sp., B. Radiofilum sp., C. Crucigeniella $\mathrm{cf}$. rectangularis, D. Pandorina sp., E. Epithemia sorex, F. Navicula sp., G. Nitzschia sp., H. Encyonema sp., I. Fragilariaceae, J. Achnanthidium.

\footnotetext{
${ }^{1}$ Laboratorio de Ecosistemas Acuáticos, Facultad de Ciencias Biológicas, Universidad Nacional Mayor de San Marcos, Lima 1, Perú, mbaylonc@unmsm.edu.pe.

${ }^{2}$ Laboratorio de Ecosistemas Acuáticos, Facultad de Ciencias Biológicas, Universidad Nacional Mayor de San Marcos, Lima 1, Perú, karenroacastro@gmail.com.

${ }^{3}$ Laboratorio de Microbiología Ambiental y Biotecnología, Facultad de Ciencias Biológicas, Universidad Nacional Mayor de San Marcos, Lima 1, Perú, tsanchezr@unmsm.edu.pe.

${ }^{4}$ Laboratorio de Acuicultura y Nutrición de Organismos Acuáticos, Facultad de Ciencias Biológicas, Universidad Nacional Mayor de San Marcos, Lima 1, Perú, ltapiaugaz@unmsm.edu.pe.

${ }^{5}$ Laboratorio de Fitología Aplicada, Facultad de Ciencias Biológicas, Código Postal 15081,Universidad Nacional Mayor de San Marcos, Lima 1, Perú, ejarap@unmsm.edu.pe.

${ }^{6}$ Laboratorio de Microbiología Ambiental y Biotecnología, Facultad de Ciencias Biológicas, Universidad Nacional Mayor de San Marcos, Lima 1, Perú, Diegomacedozero14@gmail.com.

${ }^{7}$ Laboratorio de Acuicultura y Nutrición de Organismos Acuáticos, Facultad de Ciencias Biológicas, Universidad Nacional Mayor de San Marcos, Lima 1, Perú, alansevillano@gmail.com.

${ }^{8}$ Laboratorio de Fitología Aplicada, Facultad de Ciencias Biológicas, Código Postal 15081, Universidad Nacional Mayor de San Marcos, Lima 1, Perú, abigaildextre@gmail.com.
} 